\title{
Georges-Louis Leclerc de Buffon, Histoire naturelle, générale et particulière, avec la description du Cabinet du Roi
}

\section{Cristina Dabusti}

\section{(2) OpenEdition \\ 1 Journals}

\section{Edizione digitale}

URL: https://journals.openedition.org/studifrancesi/3085

DOI: $10.4000 /$ studifrancesi.3085

ISSN: 2421-5856

\section{Editore}

Rosenberg \& Sellier

\section{Edizione cartacea}

Data di pubblicazione: 1 juillet 2013

Paginazione: 453-454

ISSN: 0039-2944

\section{Notizia bibliografica digitale}

Cristina Dabusti, «Georges-Louis Leclerc de Buffon, Histoire naturelle, générale et particulière, avec la description du Cabinet du Roi», Studi Francesi [Online], 170 (LVII | II) | 2013, online dal 30 novembre 2015, consultato il 01 février 2023. URL: http://journals.openedition.org/studifrancesi/3085 ; DOI: https:// doi.org/10.4000/studifrancesi.3085

Questo documento è stato generato automaticamente il 1 février 2023.

\section{c) (†) $\odot$}

Creative Commons - Attribuzione - Non commerciale - Non opere derivate 4.0 Internazionale - CC BYNC-ND 4.0

https://creativecommons.org/licenses/by-nc-nd/4.0/ 


\title{
Georges-Louis Leclerc de Buffon, Histoire naturelle, générale et particulière, avec la description du Cabinet du Roi
}

\author{
Cristina Dabusti
}

\section{NOTIZIA}

GEORGES-LOUIS LECLERC DE BUFFON, Histoire naturelle, générale et particulière, avec la description du Cabinet du Roi. Tome VII (1758). Texte établi, introduit et annoté par Stéphane SCHMITT avec la collaboration de Cédric CRÉMIÈRE, Paris, Honoré Champion, 2011, pp. 536.

1 Pubblicato due anni dopo il tomo VI, il volume VII dell'Historie naturelle continua il catalogo delle bestie selvatiche. Sorprendente e inatteso risulta il brusco passaggio, dopo l'analisi delle bestie selvatiche carnivore, alla descrizione dei piccoli animali nocivi per l'uomo. Ciò dà atto di una certa confusione logica e tematica che inizia a trasparire a questa altezza e che, secondo Schmitt (p. 11), caratterizzerà con sempre maggior evidenza i volumi successivi.

2 Le descrizioni, elaborate da Buffon in collaborazione con M. Dubenton, risultano più agili e rapide rispetto a quelle delle prime bestie selvatiche. Per la prima volta, inoltre, vengono riunite in un unico capitolo le informazioni ricavate dalle collezioni reali relative a due differenti tipologie animali. Tale accorpamento risponde con buona probabilità ad esigenze puramente pratiche: è possibile infatti che la descrizione del Cabinet $d u$ Roi contenesse poche notizie sugli animali meno diffusi. Il catalogo è preceduto da un lungo preambolo nel quale Buffon espone considerazioni, ardite per il contesto storico-culturale nel quale si inseriscono, sui sensi, sull'anima e sul sistema nervoso degli animali, manifestando così una certa affinità con quanto affermato dai medici vitalisti del tempo quali Jean-Joseph Ménuret e soprattutto Louis de La Caze. Gli 
animali selvatici presenti nel tomo VII sono accomunati dal fatto di non essere cacciati bensì di essere essi stessi cacciatori. Tali animali sono il lupo, la volpe, il tasso, la lontra, la faina, la martora, la puzzola, il furetto, la donnola, l'ermellino. Buffon annovera in seguito tra i piccoli animali che recano danno all'uomo lo scoiattolo, il ratto e il topo con alcuni suoi sottogeneri. Alla fine di ogni capitolo, non mancano tavole illustrate che raffigurano ogni animale sia nel suo aspetto comune sia in sezione anatomica.

Il volume VII è corredato da tre «annexes». La prima e la terza sono tratte dalle voci dell'Encyclopédie inerenti rispettivamente alle proprietà del cervello e agli animali descritti all'interno del volume. La seconda è tratta dall'Idée de l'homme physique et moral di Louis de La Caze.

4 Nell'edizione di riferimento, l'introduzione curata da Stéphane Schmitt si concentra sulla fortuna degli animali, nel corso del xviII secolo, nelle opere di carattere scientifico così come in letteratura. L'editore dà atto di una molteplicità di occorrenze letterarie delle più svariate tipologie animali nei romanzi settecenteschi, nelle nuove edizioni di opere classiche, nei resoconti di viaggio, negli scritti popolari, nei dizionari e nell'Encyclopédie, nei manuali relativi alle arti e ai mestieri, nelle opere morali e didascaliche e nei cataloghi illustrati. Tali occorrenze spaziano dalle descrizioni più naturalistiche a quelle più fantastiche. Il testo del tomo VII, modernizzato nella grafia, è supportato da note ricche e abbondanti che offrono spunti interessanti per approfondimenti soprattutto di carattere scientifico. 